\title{
Método de Papadakis e número de repetições em experimentos de soja
}

\author{
The Papadakis method and the replications number in soybean experiments
}

\author{
Lindolfo Storck ${ }^{I *}$ Alberto Cargnelutti Filho' Alessandro Dal'Col Lúcio ${ }^{I}$ \\ Sidinei José Lopes ${ }^{\mathrm{I}}$
}

\section{RESUMO}

Neste trabalho, foram utilizados dados de produtividade de grãos de 216 ensaios de genótipos de soja, realizados no Estado do Rio Grande do Sul, nos anos agrícolas 2003/2004, 2004/2005 e 2005/2006. O objetivo foi verificar se o uso do método de Papadakis reduz o número de repetições necessário à predição do desempenho de genótipos de soja em relação à produtividade de grãos. Maior precisão experimental é obtida com a aplicação do método de Papadakis, independentemente da combinação entre o tamanho de parcela e o número de repetições e a homogeneidade entre os blocos. O uso do método de Papadakis permite reduzir o número de repetições necessário à predição do desempenho de genótipos de soja, para um mesmo grau de precisão, em relação à produtividade de grãos. Ensaios com quatro repetições $e$ analisados com o método de Papadakis possibilitam a identificação de genótipos superiores de soja em relação à produtividade de grãos, com $80 \%$ de precisão.

Palavras-chave: Glycine $\max$ L., correlação intraclasse, repetibilidade, precisão experimental.

\section{ABSTRACT}

Seed yield data of 216 soybean genotype experiments conducted on of Rio Grande do Sul-Brazil during the 2002/2004, 2004/2005 and 2005/2006 were used in order to verify whether the use of Papadakis Method would decrease the number of replications necessary to predict the performance of soybean genotypes in regard to seed yield. Greater experimental precision can be obtained by using the Papadakis Method regardless the combination among plot size, number of replications and block homogeneity. The use of Papadakis Method allows reduction of replications necessary to predict seed yield performance of soybean genotypes. Experiments consisted of four replications and were analyzed according to
Papadakis Method which permitted to identify superior genotypes in regard to seed yield with $80 \%$ of precision.

Key words: Glycine max L., intraclass correlation, repeatability, experimental precision.

\section{INTRODUÇÃO}

Programas de melhoramento de soja (Glycine max L. Merrill) usam a experimentação para a identificação de genótipos superiores. Nessa etapa, é importante que características qualitativas e diferenças de produtividade de grãos entre os genótipos sejam estatisticamente significativas. A significância entre genótipos depende da existência de diferença entre eles e da magnitude do erro experimental (variância ambiental). Técnicas experimentais para a identificação de fontes de erro experimental e métodos de melhoria da precisão são relatadas na literatura (GÓMEZ \& GÓMEZ, 1984; BANZATTO \& KRONKA, 1996; STEEL et al., 1997; RAMALHO et al., 2005; STORCK et al., 2006). Especificamente, para a cultura de soja, são encontradas pesquisas para determinar o tamanho de parcela e o número de repetições adequados (MARTIN et al., 2005), a classificação da precisão experimental pelo coeficiente de variação (CARVALHO et al., 2003) e, também, a sua relação com o manejo (LÚCIO et al., 1999).

Alternativas, como diferentes métodos de análise espacial, têm sido aplicadas a fim de aumentar a

IDepartamento de Fitotecnia, Centro de Ciências Rurais (CCR), Universidade Federal de Santa Maria (UFSM), 97105-900, Santa Maria, RS, Brasil. E-mail: lindolfo@smail.ufsm.br.*Autor para correspondência. 
precisão experimental (SAEZ \& MAGAÑA, 1990; VIVALDI, 1990; AMARO et al., 1997; SOUZA et al., 2000; COSTA et al., 2005) e melhorar as estimativas dos componentes de variância e herdabilidade (COSTA et al., 2005). O método de Papadakis (PAPADAKIS, 1937) tem sido uma alternativa adequada, revelando ganhos de precisão em experimentos de competição de cultivares de milho (CARGNELUTTI FILHO et al., 2003) e soja (STORCK et al., 2008). Trata-se de um método de ajuste espacial, utilizando as médias dos erros experimentais calculadas entre as parcelas vizinhas como covariável, a fim de diminuir a variância do erro em experimentos realizados em campo. Não é conhecida a magnitude da eficiência do método de Papadakis quando relacionado com o número de repetições necessário para uma dada precisão na experimentação com soja.

As metodologias disponíveis para estimar tamanho de parcela e número de repetições dependem de experimentos específicos realizados na área experimental escolhida (STEEL et al., 1997; ALVES \& SERAPHIN, 2004; MARTIN et al., 2005), os quais nem sempre são representativos dessa mesma área nos anos seguintes devido à interação com o ambiente. Assim, 0 número de repetições, determinado por meio do método da análise de repetibilidade (CRUZ \& REGAZZI, 1997), a partir de dados de vários experimentos realizados num determinado local ou numa região, minimiza custos, aproveita as informações existentes e melhora a abrangência do plano experimental a ser usado (LIN et al., 1996).

O objetivo do estudo foi verificar se o uso do método de Papadakis reduz o número de repetições necessário à predição do desempenho de genótipos de soja em relação à produtividade de grãos.

\section{MATERIAL E MÉTODOS}

Foram usados os resultados de produtividade de grãos de soja de 216 ensaios de competição de genótipos, executados no delineamento blocos completos ao acaso com número de repetições variando entre três e quatro e de genótipos, entre 10 e 20. As unidades experimentais foram compostas por quatro filas de seis metros de comprimento e $0,4 \mathrm{~m}$ entre filas $\left(4,0 \mathrm{~m}^{2}\right.$ de área útil). Os ensaios são classificados quanto ao estágio do melhoramento (preliminares, valor de cultivo e uso e recomendados) e quanto ao ciclo (precoce, médio e tardio). Esses ensaios foram executados nos anos agrícolas 2003/2004, 2004/2005 e 2005/2006, no conjunto de áreas experimentais (diferentes locais) coordenados pela FUNDACEP (Cruz Alta, Cachoeira do Sul e Restinga Seca) e pela
FEPAGRO Sementes (Júlio de Castilhos, Santo Augusto, São Borja e Veranópolis), no Rio Grande do Sul (RS).

Em cada ensaio, foi realizada a análise de variância e foram anotados os valores do quadrado médio de blocos (QMb), do quadrado médio de genótipos (QMg), do quadrado médio do erro (QMe), da média geral ( $\widehat{m}$ ), do coeficiente de variação em percentagem da média (Cve = $100 \sqrt{ }$ QMe $/ m$ m), do número de genótipos (ng) e de repetições (J), bem como dos resultados dos testes de hipóteses para blocos e genótipos.

Foi estimado o coeficiente de correlação intraclasse para blocos $\left(\widehat{\rho}_{\mathrm{b}}\right)$ usando a expressão $\widehat{p}_{\mathrm{b}}=\widehat{\sigma}_{\mathrm{b}}^{2} /\left(\widehat{\sigma}^{2}+\widehat{\sigma}_{\mathrm{b}}^{2}\right)$, em que $\hat{\sigma}^{2}$ é o QMe e $\hat{\sigma}_{b}^{2}=(\mathrm{QMb}-\mathrm{QMe}) / \mathrm{ng}$. O índice de heterogeneidade da produção (b) de cada experimento foi estimado pela

expressão $b=1-\frac{\log \left[n g-(n g-1)\left(1-\hat{\rho}_{b}\right)\right]}{\log (n g)}$ (LIN \& BINNS, 1984).

Considerando-se as avaliações em cada bloco como medições realizadas no mesmo indivíduo (genótipo), foi estimado o coeficiente de correlação intraclasse para genótipos ou coeficiente de repetibilidade (método da análise de variância): $\hat{\rho}_{g}=\widehat{\sigma}_{g}^{2} /\left(\hat{\sigma}^{2}+\hat{\sigma}_{g}^{2}\right)$, em que $\hat{\sigma}_{\mathrm{g}}^{2}=(\mathrm{QMg}-\mathrm{QMe}) / \mathrm{J}$. O número mínimo de medições (repetições = blocos $=$ J) necessário para predizer o valor real dos indivíduos (genótipos), com base nos coeficientes de determinação $\left(R^{2}\right)$ pré-estabelecidos $\left(R^{2}=0,80\right.$ e $\mathrm{R}^{2}=0,90$ ), foi obtido conforme procedimentos de CRUZ \& REGAZZI (1997), ou seja, o valor de J para um dado $\mathrm{R}^{2}$ é $=\left(1-\hat{\rho}_{\mathrm{g}}\right) \mathrm{R}^{2} / \widehat{\rho}_{\mathrm{g}}\left(1-\mathrm{R}^{2}\right)$.

$\mathrm{Na}$ seqüência, foram estimados os valores dos erros $\left(\hat{e}_{i j}=Y_{i j}-\hat{m}-\hat{g}_{i}\right)$, considerando o delineamento inteiramente casualizado, conforme o método de PAPADAKIS (1937). Com esses valores $\left(\hat{e}_{i j}\right)$, foi estimado o valor do erro médio de cada unidade experimental como sendo a média entre o erro da unidade considerada e os erros das unidades vizinhas (lado direito, lado esquerdo, da frente e do fundo) existentes. $O$ valor do erro médio é denominado de covariável ( $\hat{C}_{i j}$ ) para fins da análise de covariância e da aplicação do método de Papadakis, segundo o delineamento inteiramente casualizado (CARGNELUTTI FILHO et al., 2003).

A análise de covariância (método de Papadakis), segundo o modelo $Y_{i j}=m+g_{i}+\beta\left(C_{i j}-\bar{C} ..\right)+\varepsilon_{i j}$, com testes de hipótese dos efeitos da covariável e de genótipos $\left(g_{i}\right)$, foi realizada conforme STEEL et al. (1997), repetindo as estimativas do coeficiente de correlação intraclasse para genótipos $\left(\hat{\rho}_{g}\right)$ e a estimativa 
do número mínimo de repetições necessário para predizer o valor real dos indivíduos (genótipos), com base nos coeficientes de determinação préestabelecidos $\left(\mathrm{R}^{2}=0,80\right.$ e $\left.\mathrm{R}^{2}=0,90\right)$. Também foi testada a hipótese do paralelismo entre os genótipos quanto à resposta da covariável (SEBER, 1976).

Para os cálculos, foi elaborado e compilado um programa específico, usando a linguagem Pascal e o aplicativo Excel.

\section{RESULTADOS E DISCUSSÃO}

Pela análise da variância, em 29,2\% dos 216 ensaios os blocos foram heterogêneos e, em 79,6\% dos ensaios, os genótipos foram significativamente diferentes quanto à produtividade de grãos. Ao aplicar o método de Papadakis, a freqüência de ensaios com diferença significativa entre genótipos passou para $98,1 \%$. Na totalidade dos ensaios, o efeito da covariável (média do erro das parcelas vizinhas) foi significativo.

A produtividade de grãos nos ensaios, que variou entre 0,44 e 3,49t ha ${ }^{-1}$ com média geral igual a $1,935 \mathrm{tha}^{-1}$ (Tabela 1), foi influenciada pela grande proporção (68,5\%) de ensaios em que são avaliadas as linhagens de primeiro, segundo ou terceiro ano, das quais a maioria será descartada nas avaliações seguintes por produtividade ou qualidade insuficientes. Quanto maior for a variação das médias dos ensaios, da amplitude entre médias de genótipos e do coeficiente de variação, maior será a abrangência de estudo e aplicação do método de Papadakis.

Sabe-se que as estimativas das médias $(\widehat{m})$ dos ensaios não são influenciadas pela aplicação do método de Papadakis (análise da covariância). No entanto, a amplitude entre as estimativas das médias, devido ao ajuste das estimativas das médias de cada genótipo em função da média da covariável, pode sofrer alterações que, no estudo, foi de baixa magnitude, independentemente da condição de homogeneidade dos blocos (Tabela 1).

Houve redução do coeficiente de variação do erro experimental (CVe) com o uso do método de Papadakis devido à diminuição do QMe, já que a $\widehat{m}$ é inalterada, com pouca influência da condição de homogeneidade dos blocos. CARGNELUTTI FILHO et al. (2003) também constataram redução do QMe e da produtividade de grãos de milho com o uso do método de Papadakis. A precisão dos 216 ensaios analisados, sem o uso do método de Papadakis, é considerada média pela classificação de LÚCIO et al. (1999), pois o valor (média dos $\mathrm{CVe}=13,3 \%$ ) está entre os limites de $9 \%$ e $15 \%$, determinados com base em 480 ensaios de avaliação da produtividade de grãos de soja no mesmo Estado (RS). Ao considerar a aplicação do método de Papadakis, a precisão continuou sendo classificada como média $(9,8 \%)$, próxima ao limite inferior dessa classe.

Tabela 1 - Valor mínimo e máximo, média, desvio padrão (dp) e coeficiente de variação (CV\%) referentes à média geral ( $\widehat{m}$, em t ha $\left.{ }^{-1}\right)$, à amplitude entre médias de genótipos (ampl) e ao coeficiente de variação do erro experimental (CVe\%) e do índice de heterogeneidade da produção (b) de 216 ensaios de avaliação de produtividade de grãos de genótipos de soja.

\begin{tabular}{|c|c|c|c|c|c|c|}
\hline \multirow{2}{*}{ Estatística } & \multicolumn{4}{|c|}{ 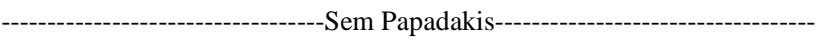 } & \multicolumn{2}{|c|}{---------Com Papadakis---------. } \\
\hline & $\hat{m}$ & ampl & $\mathrm{CVe} \%$ & $\mathrm{~b}$ & ampl & $\mathrm{CVe} \%$ \\
\hline & \multicolumn{6}{|c|}{ 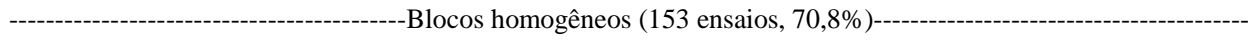 } \\
\hline Mínimo & 0,4420 & 0,3083 & 6,1 & 0,5847 & 0,3368 & 3,7 \\
\hline Máximo & 3,4889 & 1,7483 & 33,9 & 2,4191 & 1,7714 & 22,6 \\
\hline Média & 1,9595 & 0,8927 & 13,5 & 1,1227 & 0,9030 & 9,9 \\
\hline $\mathrm{dp}$ & 0,6491 & 0,3102 & 4,7 & 0,3881 & 0,3168 & 3,4 \\
\hline CV\% & 33,1 & 34,8 & 34,5 & 34,5 & 35,1 & 34,7 \\
\hline & & & os hetero & ensaios, 29, & . & \\
\hline Mínimo & 0,6097 & 0,2750 & 3,5 & 0,1947 & 0,3037 & 2,4 \\
\hline Máximo & 3,0649 & 2,0542 & 24,1 & 0,6279 & 2,0668 & 18,1 \\
\hline Média & 1,8761 & 0,8343 & 12,8 & 0,4706 & 0,8192 & 9,7 \\
\hline $\mathrm{dp}$ & 0,6039 & 0,3060 & 4,3 & 0,1212 & 0,2854 & 3,1 \\
\hline $\mathrm{CV} \%$ & 32,2 & 36,7 & 33,4 & 25,7 & 34,8 & 31,7 \\
\hline Mínimo & 0,4420 & 0,2750 & 3,5 & $\begin{array}{c}\text { saios) ---- } \\
0,1947\end{array}$ & 0,3037 & 2,4 \\
\hline Máximo & 3,4889 & 2,0542 & 33,9 & 2,4191 & 2,0668 & 22,6 \\
\hline Média & 1,9352 & 0,8757 & 13,3 & 0,9325 & 0,8786 & 9,8 \\
\hline $\mathrm{dp}$ & 0,6374 & 0,3101 & 4,6 & 0,4459 & 0,3103 & 3,3 \\
\hline CV\% & 32,9 & 35,4 & 34,3 & 47,8 & 35,3 & 33,8 \\
\hline
\end{tabular}


Sabe-se que, nos ensaios no delineamento blocos ao acaso, como neste estudo, o erro experimental é estimado como interação entre blocos e genótipos. Por isso, a média da precisão experimental (CVe) pode estar inflacionada porque $68,5 \%$ dos ensaios analisados são das classes preliminares (genótipos de diferentes ciclos no mesmo bloco), o que pode ter provocado maior interação entre estes (ciclo) e o efeito de blocos. A mistura de ciclos no mesmo bloco com efeitos ambientais distintos pode provocar a interação entre blocos e genótipos.

A média do índice de heterogeneidade da produção (b) é maior quando os blocos são homogêneos (média dos b igual a 1,1227) do que quando os blocos são heterogêneos (média dos b igual a 0,4706) (Tabela 1). Segundo LIN \& BINNS (1986), valores de b entre 0,3 e 0,7 (como no caso de blocos heterogêneos) devem ser interpretados como apropriados para redimensionar o plano experimental, variando simultaneamente o número de repetições e o tamanho de parcela. Quando b é maior do que 0,7 (como no caso dos blocos homogêneos, 70,8\% dos ensaios), é recomendado o uso de parcelas maiores para que se obtenha maior eficiência no ganho em precisão ao redimensionar o plano experimental.

Para estimar o valor da precisão $(\mathrm{D}=$ diferença significativa entre duas médias de genótipos em percentagem da média, $\mathrm{CV}=$ coeficiente de variação, $\mathrm{b}$ = índice deheterogeneidade do solo, $\mathrm{t}_{1}=$ valor tabelado de $t$ para testes de significância bilateral a 5\% de significância e $\mathrm{t}_{2}=$ valor tabelado de $\mathrm{t}$ correspondente a um erro de 2(1-P), sendo $\mathrm{P}=0,80$ a probabilidade de se obter resultados significativos), a expressão de $\operatorname{HATHEWAY~}(1961)\left(D=\sqrt{\left.2\left(t_{1}+t_{2}\right)^{2} C V^{2} / J X^{b}\right)}\right.$ mostra que o valor $\mathrm{D}$, ao redimensionar $\mathrm{J}$ (número de repetições) e X (tamanho da parcela), é mais influenciado por $\mathrm{X}$ se o valor de $\mathrm{b}$ for pequeno (menor do que 0,3 ), caso em que se recomenda usar maior J e menor X. Ao contrário, se b for próximo de um (caso dos blocos homogêneos), o uso de parcelas maiores é mais eficiente em reduzir o valor de $\mathrm{D}$. Por outro lado, ao usar $\mathrm{X}=1$, tamanho de parcela igual a uma unidade básica, o valor de $\mathrm{D}$ não depende de b. Assim, uma simulação das duas condições de blocos (homogêneos e heterogêneos), com respectivos valores de b e CV, mostram a eficiência do uso do método de Papadakis. Considerando um ensaio de $\mathrm{I}=20$ genótipos no delineamento blocos ao acaso e J repetições pode-se variar o tamanho de parcela $\left(\mathrm{X}=\right.$ unidade básica $=9,6 \mathrm{~m}^{2}$ de área total) e J, tal que a área total por tratamento seja fixa. No caso de se usar $\mathrm{J}=4$ repetições e $\mathrm{I}=20$ genótipos, o grau de liberdade do erro (GLe) é 57, e o valor crítico da distribuição de $t_{1}$ em $5 \%$ de probabilidade de erro (bilateral) ét =2,0025; $t_{2}$ é o valor da tabela de t(bilateral) correspondênte a um erro de 2(1-P), em que P é a probabilidade de serem obtidos resultados significativos (no caso, $\mathrm{P}=0,8$ ), ou seja, $\mathrm{t}_{2}=0,8480$. Assim, na tabela 2, observa-se que o uso do método Papadakis apresentou-se mais eficiente na condição de blocos homogêneos (valor de D reduziu 7,2\%) do que na condição de blocos heterogêneos (valor de D reduziu 6,2\%) para o aumento da precisão experimental com parcelas de uma unidade básica. No entanto, usando parcelas com o dobro de tamanho ( $\left.\mathrm{X}=2=19,2 \mathrm{~m}^{2}\right)$ e a metade do número de repetições, a eficiência do método de Papadakis praticamente independe da condição de homogeneidade dos blocos (Tabela 2). De qualquer forma, menores valores de D (maior precisão) são obtidos com a aplicação do método de Papadakis, independentemente da combinação entre o tamanho de parcela e número de repetições e da condição de homogeneidade dos blocos. Ganhos em precisão, com a aplicação do método de Papadakis, foram obtidos em ensaios de milho (CARGNELUTTI FILHO et al., 2003) e, segundo VIVALDI (1990), esse método é, no mínimo, de precisão semelhante se comparado com outros métodos.

Pela análise de repetibilidade, independentemente da condição de homogeneidade de blocos, o valor médio do coeficiente de correlação intraclasse ( $\hat{\rho}_{\mathrm{g}}$ ) estimado no método Papadakis foi de 0,57 , mas a redução do número de repetições necessário para uma dada precisão, com o uso do método de Papadakis, mostrou-se expressiva. Ao

Tabela 2 - Diferença significativa entre duas médias, em percentagem da média (D), de um ensaio com 20 genótipos em função da homogeneidade dos blocos, uso do método de Papadakis, respectivos coeficientes de variação (CV\%) e índice de heterogeneidade (b) para diferentes tamanhos de parcela em número de unidades básicas (X) de 9,6m².

\begin{tabular}{|c|c|c|c|c|c|c|}
\hline \multirow[b]{2}{*}{ Bloco } & \multirow[b]{2}{*}{ Papadakis } & \multirow[b]{2}{*}{$\mathrm{CV} \%$} & \multirow[b]{2}{*}{$\mathrm{b}$} & \multicolumn{3}{|c|}{--------------Tamanho de parcela (X) ------------ } \\
\hline & & & & $0,5(\mathrm{~J}=8)$ & $1(\mathrm{~J}=4)$ & $2(\mathrm{~J}=2)$ \\
\hline Homogêneos & Não & 13,5 & 1,00 & 26,9 & 27,2 & 28,2 \\
\hline Homogêneos & Sim & 9,9 & 1,00 & 19,8 & 20,0 & 20,7 \\
\hline Heterogêneos & Não & 12,8 & 0,47 & 21,3 & 25,8 & 32,1 \\
\hline Heterogêneos & Sim & 9,7 & 0,47 & 16,1 & 19,6 & 24,3 \\
\hline
\end{tabular}


aplicar o método de Papadakis, foi necessário, em média, apenas um terço do número de repetições para predizer o valor real dos indivíduos (genótipos), com base nos coeficientes de determinação préestabelecidos $\left(\mathrm{R}^{2}=0,80\right.$ e $\left.\mathrm{R}^{2}=0,90\right)$, se comparado com a análise usual (sem Papadakis) (Tabela 3).

Nos ensaios executados, em geral, são usadas quatro repetições, valor bem inferior as 12 (para blocos homogêneos) ou as 10 (para blocos heterogêneos) repetições necessárias para constatar diferenças existentes entre genótipos, com uma confiança de $80 \%$. No entanto, para uma mesma confiança, usando o método de Papadakis, as quatro repetições são suficientes, independentemente da condição de homogeneidade dos blocos.

Em 46,7\% dos ensaios, os efeitos da covariável não foram homogêneos (Tabela 3), isto é, as respostas dos diferentes genótipos à variação da covariavel não foram as mesmas, ou seja, não há paralelismo. No entanto, verifica-se que as estimativas do número de repetições têm sido muito pouco influenciadas por esse fato. Isso não quer dizer que não existam outras implicações quando se ajustam as médias de genótipos para um mesmo coeficiente (efeito da covariável) na condição de falta de paralelismo. Nesses casos, pode-se recomendar o estudo de alguma

Tabela 3 - Valor mínimo e máximo, média, desvio padrão (dp) e coeficiente de variação (CV\%) dos coeficientes de repetibilidade ( $\hat{\rho}_{\mathrm{g}}$ ) e do número de repetições $(\mathrm{J})$, associados aos diferentes coeficientes de determinação $\left(\mathrm{R}^{2}\right)$ para a avaliação de produtividade de grãos de genótipos de soja.

\begin{tabular}{|c|c|c|c|c|c|c|}
\hline \multirow[b]{2}{*}{ Estatística } & \multicolumn{3}{|c|}{-------------------Sem Papadakis------------------- } & \multicolumn{3}{|c|}{-------------------Com Papadakis------------------- } \\
\hline & $\hat{\rho}_{g}$ & $\mathrm{~J}\left(\mathrm{R}^{2}=80 \%\right)$ & $\mathrm{J}\left(\mathrm{R}^{2}=90 \%\right)$ & $\hat{\rho}_{\mathrm{g}}$ & $\mathrm{J}\left(\mathrm{R}^{2}=80 \%\right)$ & $\mathrm{J}\left(\mathrm{R}^{2}=90 \%\right)$ \\
\hline & \multicolumn{6}{|c|}{ 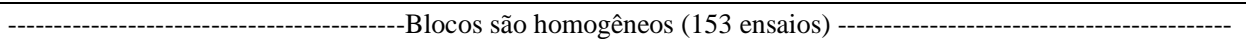 } \\
\hline Mínimo & 0,0470 & 0,9 & 2,2 & 0,2191 & 0,4 & 1,1 \\
\hline Máximo & 0,8018 & 81,1 & 182,5 & 0,8905 & 14,2 & 32,0 \\
\hline Média & 0,3726 & 12,0 & 27,1 & 0,5693 & 3,7 & 8,4 \\
\hline dp & 0,1823 & 14,5 & 32,7 & 0,1599 & 2,8 & 6,3 \\
\hline CV\% & 48,93 & 121,1 & 120,9 & 28,08 & 75,3 & 74,7 \\
\hline & \multicolumn{6}{|c|}{--------------------------------------Blocos não são homogêneos (63 ensaios) -------------------------------------- } \\
\hline Mínimo & 0,0503 & 0,8 & 1,9 & 0,1487 & 0,3 & 0,8 \\
\hline Máximo & 0,8214 & 75,5 & 169,9 & 0,9106 & 22,8 & 51,5 \\
\hline Média & 0,4078 & 9,6 & 21,6 & 0,5600 & 4,2 & 9,6 \\
\hline $\mathrm{dp}$ & 0,1940 & 11,5 & 25,8 & 0,1839 & 4,0 & 8,9 \\
\hline CV\% & 47,58 & 119,8 & 119,4 & 32,85 & 93,7 & 93,0 \\
\hline & \multicolumn{6}{|c|}{--------------------Efeito da covariável é homogêneo entre os genótipos (115 ensaios) ------------------ } \\
\hline Mínimo & 0,0488 & 0,8 & 1,9 & 0,1487 & 0,3 & 0,8 \\
\hline Máximo & 0,8214 & 77,9 & 175,4 & 0,9106 & 22,8 & 51,5 \\
\hline Média & 0,3940 & 10,6 & 23,8 & 0,5770 & 3,7 & 8,4 \\
\hline dp & 0,1966 & 12,2 & 27,5 & 0,1711 & 3,2 & 7,2 \\
\hline $\mathrm{CV} \%$ & 49,90 & 115,7 & 115,4 & 29,65 & 86,4 & 85,6 \\
\hline & \multicolumn{6}{|c|}{--------------------Efeito da covariável não é homogêneo entre os genótipos (101 ensaios) ------------------ } \\
\hline Mínimo & 0,0470 & 1,2 & 2,7 & 0,1820 & 0,4 & 1,1 \\
\hline Máximo & 0,7678 & 81,1 & 182,5 & 0,8905 & 17,9 & 40,4 \\
\hline Média & 0,3713 & 12,1 & 27,2 & 0,5558 & 4,0 & 9,0 \\
\hline dp & 0,1746 & 15,2 & 34,1 & 0,1626 & 3,1 & 7,1 \\
\hline CV\% & 47,02 & 125,7 & 125,4 & 29,25 & 78,8 & 78,2 \\
\hline Mínimo & 0,0470 & 0,8 & 1,9 & $\begin{array}{l}\text { os) --.-- } \\
0,1487\end{array}$ & 0,3 & 0,8 \\
\hline Máximo & 0,8214 & 81,1 & 182,5 & 0,9106 & 22,8 & 51,5 \\
\hline Média & 0,3829 & 11,3 & 25,5 & 0,5666 & 3,9 & 8,7 \\
\hline dp & 0,1865 & 13,8 & 31,0 & 0,1673 & 3,2 & 7,2 \\
\hline CV\% & 48,71 & 121,79 & 121,51 & 29,53 & 82,59 & 81,93 \\
\hline
\end{tabular}


forma de ajuste das médias por estratificação de genótipos.

\section{CONCLUSÕES}

O uso do método de Papadakis permite reduzir o número de repetições necessário para a predição do desempenho de genótipos de soja, para um mesmo grau de precisão, em relação à produtividade de grãos.

Ensaios com as usuais quatro repetições analisados com o método de Papadakis possibilitam a identificação de genótipos superiores de soja em relação à produtividade de grãos, com 80\% de precisão.

\section{AGRADECIMENTOS}

Ao Conselho Nacional de Desenvolvimento Científico e Tecnológico (CNPq), pela concessão de bolsa de produtividade aos autores, e aos pesquisadores que auxiliaram os autores na realização dos experimentos, pela cessão dos dados.

\section{REFERÊNCIAS}

ALVES, S.M.F.; SERAPHIN, J.C. Coeficiente de heterogeneidade do solo e tamanho de parcela. Pesquisa Agropecuária Brasileira, v.39, p.105-111, 2004. Disponível em: <http://www.scielo.br/ s c i e lo.ph p ? s c ri pt = sci_art text \& pid = S $0100-$ 204X2004000200002\&lng=en\&nrm=iso\&tlng=pt $>$. Doi: 10.1590/ S0100-204X2004000200002.

AMARO, R. et al. Comparacion de metodos de ajuste en el analisis espacial de experimentos de campo. Agronomia Tropical, v.47, n.3, p.359-374, 1997.

BANZATTO, D.A.; KRONKA, S.N. Experimentação agrícola. Jaboticabal: FUNEP, 1996. 247p.

CARGNELUTTI FILHO, A. et al. Ajustes de quadrado médio do erro em ensaios de competição de cultivares de milho pelo método de Papadakis. Pesquisa Agropecuária Brasileira, v.38, p.467-473, 2003. Disponível em: <http://www.scielo.br/

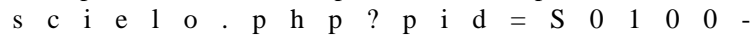
204X2003000400004\&script $=$ sci_arttext \&thng $=p t>$. Doi: 10.1590/S0100-204X2003000400004.

CARVALHO C.G.P. et al. Proposta de classificação dos coeficientes de variação em relação à produtividade e altura da planta de soja. Pesquisa Agropecuária Brasileira, v.38, n.2, p.187-193, 2003. Disponível em: http://www.scielo.br/scielo.php?pid=S0100204X2003000200004\&script=sci_arttext\&tlng=pt. Doi: 10.1590/ S0100-204X2003000200004.

COSTA, J.R. et al. Análise espacial e de vizinhança no melhoramento genético de plantas. Pesquisa Agropecuária Brasileira, v.40, n.11, p.1073-1079, 2005. Disponível em: http:/ /www.scielo.br/scielo.php?script=sci_arttext\&pid=S0100204X2005001100004\&lng=en\&nrm=iso\&tlng=pt. Doi: 10.1590/ S0100-204X2005001100004.

CRUZ, C.D.; REGAZZI, A.J. Modelos biométricos aplicados ao melhoramento genético. 2.ed. Viçosa: UFV, 1997. 390p.
GÓMEZ, K.A.; GÓMEZ, A.A. Statistical procedures for agricultural research. 2.ed. New York: John Wiley, 1984. $680 p$.

HATHEWAY, W.H. Convenient plot size. Agronomy Journal, v.53, p.279-280, 1961.

LIN, C.S. et al. Persistence of a field heterogeneity index. Canadian Journal of Plant Science, v.76, p.245-250, 1996.

LIN, C.S.; BINNS, M.R. Relative efficiency of two randomized block designs having different plot size and numbers of replications and of plots per block. Agronomy Journal, v.78, p.531-534, 1986.

LIN, C.S.; BINNS, M.R. Working rules for determining the plot size and numbers of plots per block in field experiments. Journal of Agricultural Science, v.103, p.11-15, 1984.

LÚCIO, A.D. et al. Classificação dos experimentos de competição de cultivares quanto a sua precisão. Pesquisa Agropecuária Gaúcha, v.5, n.1, p.99-103, 1999.

MARTIN, T.N. et al. Tamanho ótimo de parcela e número de repetições em soja (Glycine max (L.) Merril). Ciência Rural, v.35, p.271-276, 2005. Disponível em: http://www.scielo.br/ s c i e l o.ph p ? s c ri p t = s c i_art text \& pi d = S 0103 $84782005000200004 \& \operatorname{lng}=$ en\&nrm=iso\&tlng=pt. Doi: 10.1590/ S0103-84782005000200004.

PAPADAKIS, J.S. Méthode statistique pour des expériences sur champ. Thessalonike: Institut d’Amélioration des Plantes à Salonique, 1937. 30p. (Bullettin, 23).

RAMALHO, M.A.P. et al. Experimentação em genética e melhoramento de plantas. 2.ed. Lavras: UFLA. 2005. 322p.

SAEZ, T.C.; MAGAÑA, J.A.J. El análisis de experimentos por método Papadakis. Chapingo, v.15, n.71-72, p.110-113, 1990 .

SEBER, G.A.F. Linear regression analysis. NewYork: John Wiley \& Sons, 1976. 465p.

SOUZA, E.A. et al. Alternativas experimentais na avaliação de famílias em programas de melhoramento genético de feijoeiro. Pesquisa Agropecuária Brasileira, v.35, n.9, p.1765-1771, 2000.

STEEL, R.G.D. et al. Principles and procedures of statistics: a biometrical approach. 3.ed. New York: McGraw Hill Book, 1997. 666p.

STORCK, L. et al. Utilização do método de Papadakis na melhoria da qualidade experimental dos ensaios de soja. Pesquisa Agropecuária Brasileira, v.43, n.5, p.581-587, 2008. Disponível em: http://www.scielo.br/scielo.php?pid=S0100204X2008000500005\&script=sci_arttext. Doi: 10.1590/S0100204X2008000500005.

STORCK, L. et al. Experimentação vegetal. 2.ed. Santa Maria: UFSM, 2006. 198p.

VIVALDI, L.J. Comparação entre métodos de análise espacial de experimentos de campo. Pesquisa Agropecuária Brasileira, v.25, n.1, p.77- 84, 1990. 